\title{
Evaluation of microcapillary culture method for the isolation of Leishmania aethiopica parasites from patients with cutaneous lesions in Ethiopia
}

\author{
Lensa Aberra ${ }^{1}$, Adugna Abera ${ }^{2 *}$, Tariku Belay ${ }^{3}$, Amha Kebede $^{2}$, Endalamaw Gadisa ${ }^{1}$ and Geremew Tasew ${ }^{2}$
}

\begin{abstract}
Background: In addition to direct slide microscopy, traditional culture method (TCM) has long been considered as a gold standard method for the diagnosis of cutaneous leishmaniasis (CL). However, TCM is relatively expensive and time-consuming compared to the newly introduced microculture method (MCM), which has shown to be sensitive and rapid diagnostic method elsewhere for different Leishmania parasite species other than Leishmania (L.) aethiopica. The objective of this study was to evaluate the diagnostic performance of $\mathrm{MCM}$ for the diagnosis of $\mathrm{CL}$ caused by L. aethiopica.

Methods: One hundred forty-three lesion aspirates were collected from 124 suspected $C L$ patients prospectively based on their consecutive series. Portion of the aspirates were cultured in duplicate in TCM with modified NovyMacNeal-Nicolle (NNN) in tissue culture flask and microcapillary tubes containing RPMI 1640 with 10\% fetal bovine serum (FBS) for MCM. Smears on glass slides from the remaining portion of the aspirate were used for direct microscopy to detect the parasite after stained with Giemsa staining solution. Up on a consensus, positive result in any two of the three tests was used as a reference standard to analyze sensitivity.

Results: As per consensus standard criteria, 52 of the lesions were qualified to evaluate MCM versus TCM. Fortyeight lesion samples were positive by MCM, 36 by TCM, and 37 by smear microscopy. The representative DNA from parasite culture isolates revealed the causative Leishmania parasite was L. aethiopica by ITS1 polymerase chain reaction-restriction fragment length polymorphism (PCR-RFLP). Culturing L. aethiopica in vitro by MCM is more sensitive (92.3\%) than by TCM (69.2\%), $P=0.003$. The median time for $L$. aethiopica promastigotes emergence in the culture was 3 days for MCM and 6 days for TCM, $P \leq 0.001$.

Conclusions: Our finding indicated that MCM is a sensitive and a rapid culturing method for the isolation of $L$. aethiopica than TCM and smear microscopy.
\end{abstract}

Keywords: Traditional culture method, ITS1-PCR-RFLP, NNN, Diagnosis of CL

\footnotetext{
* Correspondence: adugna.abera2020@gmail.com

${ }^{2}$ Ethiopia Public Health Institute, Malaria and Neglected Tropical Diseases

Research Team, P. O. Box 1242, Addis Ababa, Ethiopia

Full list of author information is available at the end of the article
}

C The Author(s). 2019 Open Access This article is distributed under the terms of the Creative Commons Attribution 4.0 International License (http://creativecommons.org/licenses/by/4.0/), which permits unrestricted use, distribution, and reproduction in any medium, provided you give appropriate credit to the original author(s) and the source, provide a link to the Creative Commons license, and indicate if changes were made. The Creative Commons Public Domain Dedication waiver (http://creativecommons.org/publicdomain/zero/1.0/) applies to the data made available in this article, unless otherwise stated. 


\section{Introduction}

Leishmaniasis is a vector-borne disease caused by various species of Leishmania parasites which are transmitted to mammalian host by the bite of female sand fly. The disease is expressed by various clinical manifestations ranging from self-healing cutaneous lesions to potentially fatal visceral form [1]. Cutaneous leishmaniasis (CL) is a disfiguring skin disease, with the potential of long-term psychological and social consequences, especially in young women [2]. In Ethiopia, the CL form of the disease has got three clinical forms such as localized (LCL), mucocutaneous (MCL), and diffuse CL (DCL) [3].

Cutaneous leishmaniasis is the commonest form of leishmaniasis in Ethiopian highland which is predominantly caused by Leishmania $(L)$. aethiopica. It is becoming a growing public health concern with increased number of cases and new outbreaks in areas of previously not known to be endemic [4]. It is a zoonotic disease in which the parasites in the ecological system is maintained by rock hyraxes species of Procavia capensis and Heterohyrax brucei, that have been incriminated as the only known reservoir hosts of L. aethiopica [5].

The diagnosis of CL in endemic areas can be made on the basis of clinical and epidemiological data. However, due to potential toxicity associated with standard pentavalent antimonial therapy, identification of the parasites in the clinical specimens is important. Thus, timely and definitive diagnosis is important for the appropriate management of CL. The definitive diagnosis of CL includes visualization of the amastigotes by microscopic examination of Giemsa stained smears or in histological sections and in vitro culture of the parasite [6]. Although microscopic examination is rapid, cheap, and easy to perform, it lacks sensitivity due to the generally low number of parasites found in tissue samples [7]. In vitro cultures obtained from aspirates, biopsies, or from skin scrapings are reported to be more sensitive than microscopy, but the sensitivity is variable and the differences are based on various factors as for example, the viability of collected parasites, the strain and the media used, the presence of super infection, and the expertise of the investigator [8]. Polymerase chain reaction (PCR) is considered as the most sensitive method for the diagnosis of CL [9-13]. However, this method is not yet available outside of the research setting and still remains expensive for field operation. The microculture method (MCM) is reported to be sensitive and has less promastigote emergence time than traditional culture method (TCM) for the diagnosis of CL as described elsewhere [11-16] for CL caused other than L. aethiopica.

Cutaneous leishmaniasis induced by L. aethiopica is clinically diverse and pleotropic with high genetic diversity as well as resistant to most standard anti-leishmaniasis drugs [17]. Due to such diversity, diagnostic tools working out for other CL may or may not work for the detection of CL due to L. aethiopica. Therefore, the objective of this work was to evaluate MCM for isolation of $L$. aethiopica parasite from cutaneous lesions in Ethiopia.

\section{Materials and methods \\ Study area and period}

The study was conducted in three health centers (Ankober, Kela, and Kibet) and at All African Leprosy, Tuberculosis and Rehabilitation Training (ALERT) Hospital from April 2012 to February 2013. Ankober health center is located in Amhara Regional State, which serves for 3 towns and 19 administrative kebeles (lowest administrative unit). Kela and Kibet health centers are located in Southern People Nations and Nationalities Regional State. ALERT Hospital is located in Addis Ababa at $7 \mathrm{~km}$ southwest. Cutaneous leishmaniasis patients are referred to this hospital from almost all over the country in which they get diagnosis as well as treatment services both at the outpatient and inpatient departments.

\section{Study population}

Patients who were referred to the health center or hospital for suspected CL had a clinical indication for skin scraping or aspirate and were able to provide informed consent before included into the study. Children less than 5 years of age, patients with lesions indicative of inter-current bacterial or fungal super-infection and patients on active treatment for CL were excluded from the study. The operational definition for CL was fulfilled when clinical description that involves appearance of one or more lesions with duration of $\geq 2$ weeks, typically on uncovered parts of the body such as the face, neck, arms, and legs were enrolled [5].

Consensus reference standard: defined when a lesion was positive by any two of the three tests (MCM, TCM, and smear microscopy). These tests were served as "reference" standard against which an index test was compared.

\section{Sample size calculation}

Sample size was determined as described previously [11]. The sensitivity and the median time of positivity of TCM and MCM were estimated to be $56 \%$ (5.6 \pm 0.5 days) and $75 \%$ ( $3.5 \pm 0.5$ days $)$ respectively. In order to detect increment in sensitivity of MCM and significant difference in time of positivity, $\alpha=0.05$ and power of $80 \%$ was assumed to calculate the number of lesions using two population proportion formulas as follows:

$\mathrm{z}_{1-\alpha}=$ standard normal $z$ values corresponding to the selected alpha

$\mathrm{z}_{1-\beta}=$ standard normal $z$ values corresponding to the selected beta 
$P=$ simple average of the expected proportions $P 1$ and $P 2=$ expected sensitivity of each method (TCM and MCM)

$n=$ number of lesions required in each groups

$$
\begin{aligned}
n & =\frac{\left\{z_{1-\alpha} \sqrt{[2 P(1-P)]}+\mathrm{z}_{1-\beta} \sqrt{[P 1(1-P 1)+P 2(1-P 2)]}\right\}^{2}}{(P 1-P 2) 2} \\
& =\frac{[1.65 \sqrt{[2 \times 0.65(0.35)]}+0.84 \sqrt{[0.56(0.44)+0.75(0.25)]}]^{2}}{(0.75-0.56) 2}
\end{aligned}
$$$$
+10 \% \text { of } n=99
$$

Given the above assumptions, the total number of lesions required was calculated to be 99 . However, we have collected samples from a total of 143 lesions to compensate for those lesions which were difficult to collect all the three samples specified.

\section{Clinical sample collection}

Each cutaneous lesion of study participants were physically examined by dermatologist for proper identification of the lesion and exclusion of super infection. The lesion aspirates were collected by experienced nurse from individuals who were clinically suspected for CL. Aseptically, lesion aspirates were collected using a 25-gauge needle and disposable syringe containing $0.5 \mathrm{ml}$ of sterile saline $(85 \% \mathrm{NaCl}, \mathrm{pH}=7)$ which was inserted intra-dermally into the outer border of the lesion. The syringe was rotated, and then tissue fluid was gently aspirated into the needle as it is withdrawn. The aspirated fluid was placed in sterile cryotubes which was transported to Armauer Hansen Research Institute (AHRI) Leishmaniasis laboratory from the field sites within cold chain. The aspirated material was divided equally under sterile condition which was then inoculated in TCM or MCM within 5$10 \mathrm{~h}$ after collection. Portion of the aspirate at the edge of the needle was used to make a smear on clean glass slide for direct microscopy.

\section{Direct amastigote detection}

Air dried smear on glass slide were fixed by methanol and stained with $10 \%$ Giemsa for 25 min. Slides were examined under light microscopy with $\times 100$ objective for detection and quantification of the burden of amastigotes form based on Chulay and Bryceson method [18]. All slides were examined prior to the knowledge of the culture results to avoid subjective interpretation of results and $10 \%$ of the slides were confirmed by independent laboratory personnel as part of quality control.

Traditional culture method was performed by using Novy-MacNeal-Nicolle (NNN) medium and Locke's solution as an overlay media. Whereas for MCM, a commercially available liquid media RPMI 1640 medium
(Sigma Chemical Co.) was used with $2 \mathrm{mM}$ L-glutamine and $25 \mathrm{mM}$ Hepes (Sigma Chemical Co.) which was supplemented with 15-20\% fetal bovine serum (FBS) (Sigma Chemical Co.) and $100 \mathrm{U} / 100 \mu \mathrm{g} / \mathrm{ml}$ penicillin-streptomycin (Sigma Chemical Co.). The $\mathrm{pH}$ solution was adjusted to 7.2 and filtered through $0.2 \mu \mathrm{m}$ pore size diameter filter.

\section{Culturing techniques}

Aspirated fluid was inoculated in TCM and the MCM in duplicate and parallel inside bio-safety cabinet under sterile condition. For TCM, 150-200 $\mu \mathrm{L}$ aspirated fluid was inoculated on NNN media immediately after the 3 $\mathrm{ml}$ of Locke's solution was dispensed in the media. The inoculated culture flasks were incubated at $25-26^{\circ} \mathrm{C}$. Whereas MCM was performed by mixing $150-200 \mu \mathrm{L}$ lesion aspirates with equal volume of complete RPMI 1640 medium in sterile cryo-tube. The mixture was then loaded $2 / 3$ length of $1 \times 75 \mathrm{~mm}$ non-heparinized microhematocrit capillary tubes (Heinz, Germany) using $1 \mathrm{ml}$ syringe as described previously [19]. The ends of the capillary tubes were sealed and incubated horizontally at $25-26{ }^{\circ} \mathrm{C}$ temperature. All inoculated culture flasks and microcapillary tubes were examined every day under an inverted microscope (Leitz-Wetzlar, Germany) with $\times 20$ and $\times 40$ objectives for observation of motile promastigotes in the culture. Cultures were considered positive when a motile promastigote was observed and negative if there was no motile promastigote after being examined for 30 days. The isolates grown to late exponential growth phase were used for DNA extraction and subsequent PCR-RFLP species identification.

\section{DNA extraction and species typing}

The genomic DNA was extracted from culture isolates using commercial kit (QIAamp DNA Mini Kit; Qiagen, Chatsworth, CA, USA) in accordance with the manufacturer's instructions. Species typing was done using the internal transcriber spacer-1 (ITS1)-RFLP as described by Schonian et al. [20] and Gadisa et al. [21].

\section{Data processing and statistical analysis}

Descriptive statistics was calculated for continuous variables. Categorical variables were quantified by proportions, and statistical analyses were performed using STATA version 11 (StataCorp LP, College Station, TX). The sensitivity, specificity, positive, and negative predictive values of the tests was calculated by using the consensus standard (positive results of any two of three tests) as the "reference standard." Differences in time to culture positivity were compared between groups by using one-way analysis of variance. Differences in sensitivities were compared using the $z$ test. The agreement 
between two tests was assessed by kappa value, and the level of significance was set as a $P<0.05$.

\section{Quality assurance}

To check the quality of culture media, positive control cultures was inoculated and treated as the same manner as the clinical isolates. Culture medium with no clinical sample was also used as negative controls. Reference strains (L. aethiopica (MHOM/ET/72/L100), L. donovani (MHOM/IN/80/DD8), L. chagasi (MHOM/BR/00/1669), L. infantum (MHOM/FR/LEM-75), L. tropica (MHOM/ $\mathrm{SU} / 74 / \mathrm{K} 27$ ), and L. major (MHOM/ SU/73/5-ASKH) were used as positive controls for species identification and PCR amplification.

\section{Results}

A total of 124 suspected CL cases, of which $38,33,38$, and 15 of the CL patients were recruited from ALERT Hospital, Ankober, Kela, and Kibet health centers respectively (Fig. 1). Seventy-eight of the study participants were males. From these 124 suspected CL cases, 143 suspected skin lesions were collected and considered as unit of analysis.

The median duration of lesion was 9 months (range 1 month to 20 years). Clinical observation revealed that out of the 143 lesions, 95 (66.4\%) were suspected to be LCL, whereas the rest 37 (25.9\%) and 11 (7.7\%) were assumed to be MCL and DCL respectively. Seventy-six (53.1\%) of the 143 lesions were ulcerated while the rest 67 (46.9\%) were nodular lesions.

Considering the consensus standard criteria as a reference standard, 52 out of 143 lesions fulfilled the criteria for the diagnosis of CL. Sixty-three lesion samples were positive by at least one of the three tests and 25 were positive by three tests. Of the 52 lesions that were

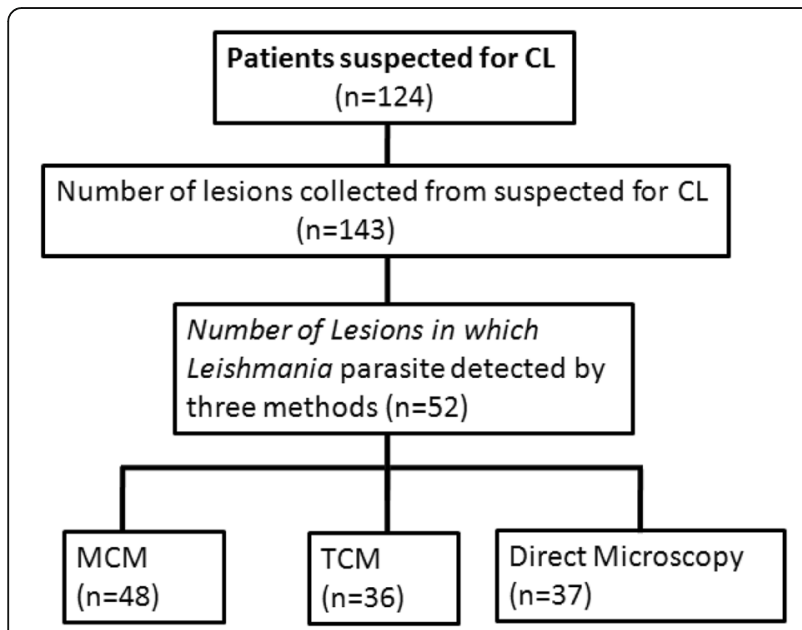

Fig. 1 Flow diagram for diagnostic evaluation of MCM for isolation of $L$. aethiopica from $C L$ lesions in Ethiopia positive by standard criteria, all of them were culture positive by MCM and/or TCM. From these, 48 of the lesions were positive by MCM and 36 of the lesions were positive by TCM, while 37 lesions were positive by direct microscopy (Table 1).

From the standard consensus criteria, $76.9 \%$ of positive lesions were clinically suspected for LCL $(P \leq 0.001)$, of which $90 \%$ were also positive by MCM. When results are compared across lesion appearance, majority of positive lesions were non-ulcerative and the difference was statistically significant $(p=0.013)$.

The overall sensitivity and specificity of MCM was 92.3\% (95\% $[\mathrm{CI}]=84.8-99.8 \%)$ and $97.8 \%$ respectively. The sensitivity and specificity of TCM was $69.2 \%$ (95\% $[\mathrm{CI}]=54.1-84.3 \%)$ and $98.9 \%$ respectively $(P=0.003)$ (Table 2). The agreement between MCM and TCM was $83.9 \%$ with kappa value of $0.642(P \leq 0.001)$. From Table 1 above, it is clear that isolation of $L$. aethiopica parasite from non-ulcerative LCL with lesion duration less than 12 months were best recovered by MCM than TCM and direct microscopy.

When individual patient was used as unit of analysis, the sensitivity of MCM and TCM was $91.1 \%$ and $71.1 \%$ respectively, while specificity for both MCM and TCM was 98.6\%; which did not change substantially from the per-lesion analysis and remained statistically significant $(P=0.013)$.

Thirty-seven lesions were positive by direct microscopy which yielded a sensitivity of $71.2 \%(95 \%[\mathrm{CI}]=$ $54.5-80 \%)$. There was a moderate agreement $(\mathrm{kappa}=$ 0.430 ) between smear and TCM whereas a substantial agreement $(\mathrm{kappa}=0.671)$ was found between smear and MCM $(P=0.0018)$.

Even though MCM was more sensitive (92.3\%) than TCM (69.2\%) and direct microscopy $(71.2 \%)$ in isolation of $L$. aethiopica, all the three tests have similar specificity (Table 2). The three methods have similar in respective of positive predictive value, but MCM (95.7\%) has more negative predictive value than the other two methods (Table 2).

\section{Amastigote to promastigotes transformation is faster in MCM than in TCM}

The median time to culture positivity for MCM was 3 days (range 2-11 days) and 6 days for TCM (317 days) $(P \leq 0.001)$. When the individual patient was used as unit of analysis, median time to culture positivity did not change substantially from the per-lesion analysis and remained statistically significant (median $=3$, range $2-10$ ) while it was 5.5 (range $=3-17$ ) by TCM (Table 3 ).

We also compared the costs of MCM and TCM in terms of media consumption per test; sheep blood requirement, autoclaving, and requirement for distilled water. As indicted in Table 4, MCM uses $75 \mu \mathrm{L}$ of medium per test compared to TCM which uses $4000 \mu \mathrm{L}$ 
Table 1 Clinical characteristics of confirmed CL lesions ( $n=52)$ among 143 suspected lesions by CL types, appearance, and lesion duration

\begin{tabular}{|c|c|c|c|c|c|}
\hline \multirow{2}{*}{$\begin{array}{l}\text { Clinical } \\
\text { characteristics }\end{array}$} & \multirow{2}{*}{$\begin{array}{l}\text { Total no. } \\
\text { of lesion }\end{array}$} & \multicolumn{3}{|c|}{ Positive parasitological methods } & \multirow{2}{*}{$\begin{array}{l}\text { Confirmed } \\
\mathrm{CL} \text { lesions, } \\
\mathrm{n}(\%)\end{array}$} \\
\hline & & MCM, n (\%) & TCM, n (\%) & Microscopy, n (\%) & \\
\hline \multicolumn{6}{|l|}{ CL types } \\
\hline $\mathrm{LCL}$ & $95(66.4)$ & $36(69.2)$ & $26(50)$ & $29(55.7)$ & $40(76.9)^{* *}$ \\
\hline $\mathrm{MCL}$ & $37(25.9)$ & $3(5.8)$ & $3(5.8)$ & $1(1.9)$ & $3(5.8)$ \\
\hline $\mathrm{DCL}$ & $11(7.7)$ & $9(17.3)$ & $7(13.5)$ & $7(13.5)$ & $9(17.3)$ \\
\hline Total & $143(100)$ & $48(92.3)$ & $36(69.2)$ & $37(71.1)$ & $52(100)$ \\
\hline \multicolumn{6}{|l|}{ CL appearance } \\
\hline Ulcerative & $76(53.1)$ & $17(32.7)$ & $13(25)$ & $12(23.1)$ & $20(38.5)$ \\
\hline Non ulcerative & $67(46.9)$ & $31(59.6)$ & $23(44.2)$ & $23(44.2)$ & $32(61.5)^{*}$ \\
\hline Total & $143(100)$ & $48(92.3)$ & $36(69.2)$ & $35(67)$ & $52(100)$ \\
\hline \multicolumn{6}{|l|}{ Lesion duration } \\
\hline$\leq 12$ months & $108(75.5)$ & $38(73.1)$ & $28(53.8)$ & $25(48.1)$ & $28(53.8)$ \\
\hline$>12$ months & $35(24.5)$ & $12(23.1)$ & $9(17.3)$ & $11(21.1)$ & $24(46.2)$ \\
\hline Total & $143(100)$ & $50(96.2)$ & $37(71.1)$ & $36(69.2)$ & $52(100)$ \\
\hline
\end{tabular}

${ }^{* *} P \leq 0.001$ and ${ }^{*} P \leq 0.01$. DCL, diffuse cutaneous leishmaniasis; $M C L$, mucocutaneous leishmaniasis; $L C L$, localized cutaneous leishmaniasis

sheep blood and $2000 \mu \mathrm{L}$ Locke's medium per test. The cost for $100 \mathrm{ml}$ of complete RPMI medium is approximately 4.5 USD and the cost of $100 \mathrm{ml}$ Locke's semi-solid medium is approximately 1 USD. From this comparison, the cost of MCM is at least 5.9 times cheaper than that of TCM.

\section{Species typing by PCR-RFLP confirms that the infecting parasites were L. aethiopica}

Amplification by the ITS-1 primer pairs produced PCR product of about 328 bp (Fig. 2a). When the PCR product was digested by Hha I enzyme, $L$. aethiopica reference strain and the DNA isolates from cultured promastigotes formed similar bands approximately 162 bp size (Fig. 2b). This confirms the infecting Leishmania parasites were L. aethiopica. L. major on the other hand yielded two bands of about $88 \mathrm{bp}$ and $240 \mathrm{bp}$ while, L. infantum, L. donovani, L. chagasi, and L. tropica gave single band size of $328 \mathrm{bp}$.

\section{Discussion}

The conventional CL diagnostic methods currently employed in Ethiopia are slide microscopy and cultivation of Leishnamia parasite using TCM. However, utilization of slide microscopy is compromised by lesser sensitivity while TCM is not considered applicable in regional laboratories due to logistic and infrastructure barriers. In present study, we have set a consensus standard criteria in which any two positive tests out of the three tests were considered as a gold standard against which each individual method is compared [22]. In isolation of Leishmania promastigotes from CL lesions in the present study, MCM was shown to be more sensitive (92.3\%) than the TCM (69.2\%) and smear microscopy (71.1\%). Microculture method is offering a simpler, cost-effective, and sensitive alternative to TCM and smear microscopy in the diagnosis of CL. In the previous studies, the sensitivity of TCM shown to be varied with parasites load [12], culture media used [16], Leishmania species [23] duration, and appearance of lesions [15]. In contrast, the sensitivity of MCM was shown not to be affected by CL clinical categories [15]. The higher sensitivity of MCM in the present report is in agreement with the previous finding (83.3-97\%) by Allahverdiyev et al., who also explained the reason to be due to the capillary tube ability to concentrate the sample material and

Table 2 Comparison of performance of three methods for diagnosis of CL from suspected lesions against consensus standard (gold standard, positive $=52$, negative $=91$ )

\begin{tabular}{lllllll}
\hline Diagnostic method & No. of positive & No. of negative & Sensitivity (\%) & Specificity (\%) & PPV (\%) & NPV (\%) \\
\hline Direct microscopy & 37 & 106 & 71.2 & 97.8 & 94.6 & 84.0 \\
MCM & 48 & 95 & $92.3^{+\dagger}$ & 97.8 & 96.0 & 95.7 \\
TCM & 36 & 107 & 69.2 & 98.9 & 97.3 & 84.9 \\
\hline
\end{tabular}

${ }^{+} P=0.003$ versus $\mathrm{TCM}$ by $z$ test, ${ }^{\dagger} P=0.0018$ versus direct microscopy by $z$ test 
Table 3 Time to transformation of amastigotes to promastigotes by culture methods

\begin{tabular}{|c|c|c|c|c|c|c|}
\hline \multirow{2}{*}{$\begin{array}{l}\text { Culturing } \\
\text { method }\end{array}$} & \multicolumn{3}{|c|}{ Time to positivity in days (per lesion analysis) } & \multicolumn{3}{|c|}{ Time to positivity in days (per patient analysis) } \\
\hline & Mean \pm SD & Median & Range & Mean \pm SD & Median & Range \\
\hline$\overline{M C M}$ & $3.7 \pm 1.9$ & $3^{*}$ & $2-11$ & $3.4 \pm 1.6$ & 3 & $2-10$ \\
\hline TCM & $5.8 \pm 2.5$ & 6 & $3-17$ & $5.8+2.6$ & 5.5 & $3-17$ \\
\hline
\end{tabular}

${ }^{*} P \leq 0.001$ by median test

provide microaerophilic conditions favorable for transformation of the amastigotes to promastigotes. In addition, higher partial $\mathrm{CO}_{2}\left(\mathrm{pCO}_{2}\right)$ in $\mathrm{MCM}$ was reported in the same study which corresponds with the reduction in $\mathrm{pO}_{2}$ and $\mathrm{pH}$ also favoring the survival of promastigotes [12].

Nevertheless, other studies in Peru reported lesser MCM sensitivity than our finding which is $71.7 \%$ [11] and $78.3 \%$ [15]. This variation could be attributed to the difference in Leishmania species involved in which in Peru the characterized parasite was $L$. (V.) braziliensis complex, different from ours, L. aethiopica. However, the lesser median time of MCM positivity reported in our case was in agreement with previous studies, and there is no considerable difference was observed among species [15].

MCM have been shown to be rapid and easy for transportation, and characterization of microcapillary cultivated promastigotes of $L$. donovani [19], L. tropica, and L. infantum [24]. In the present study, eight MCM (two from each study sites) were mass cultured and characterized by ITS1-RFLP which were identified to be $L$. aethiopica.

Since there is no safe procedure for extraction of cultured promastigotes, we have adapted our own techniques which involve breaking the capillary tubes with care and withdrawing the fluid with fine needle. However, such maneuver has to be evaluated further with care to minimize the risks of laboratory acquired leishmania infection.

In terms of medium consumption, MCM uses $75 \mu \mathrm{L}$ of medium per test compared to TCM which uses $2000 \mu \mathrm{L}$ medium per test. Similarly, the costs of MCM is at least 5.9 times cheaper than that of TCM, since the cost of $100 \mathrm{ml}$ of complete RPMI medium is approximately 4.5 USD and the cost of $100 \mathrm{ml}$ Locke's semi-solid medium is approximately 1 USD.

A $100 \mathrm{ml}$ sheep blood and $50 \mathrm{ml}$ of Locke's overlay solution could be used for $20-25$ tests at a total cost of 10.5 USD. Whereas, a $100 \mathrm{ml}$ of complete media could be used for approximately 1333 tests at a total cost of 4.5 USD [25]. Microculture method could not occupy much space in the incubator and easy to transport from place to place compared to TCM. One of the most advantages of $\mathrm{MCM}$ is that it can utilize light microscope which could be available at all district levels compared to TCM which utilizes inverted microscope which is only available at higher institutions.

Rapid isolation of CL causative organism in Ethiopia will also facilitate more rapid species identification in institutions where molecular diagnostic capabilities exist. Because the experience from other countries shows that species identification becomes increasingly important due to species variation in response to therapy [26] and in a region where leishmainasis/HIV co-infection is considered high [27]. Inter-specific variability in response to standard anti-leishmaniasis drugs, such as amphotericin $\mathrm{B}$, miltefosine, pentamidine, and paromomycin, has been observed among other species of Leishmania parasites somewhere else [26]. In respective of these views, prompt diagnosis and rapid isolation with species identification of CL in Ethiopia are so clinically helpful for dermatologists to treat $\mathrm{CL}$ and may contribute in the control and prevention of the disease.

Table 4 Comparison of MCM and TCM media in terms of costs

\begin{tabular}{lll}
\hline Comparison & MCM & TCM \\
\hline Cost of $100 \mathrm{ml}$ sheep blood & Not required & 10 USD \\
Filtration through $0.22 \mathrm{~mm}$ & Required & Required for Locke's solution \\
Media required/test & $75 \mu \mathrm{L}$ complete media & $4 \mathrm{ml}$ sheep blood and $2 \mathrm{ml}$ Locke's solution \\
Cost of Locke's solution/100 ml & Not required & 1 USD \\
Autoclaving & Not required & Required \\
Distilled water & Not required & Required \\
\hline
\end{tabular}

The data for comparison was collected from store records, local shops, pharmacy, and laboratories 


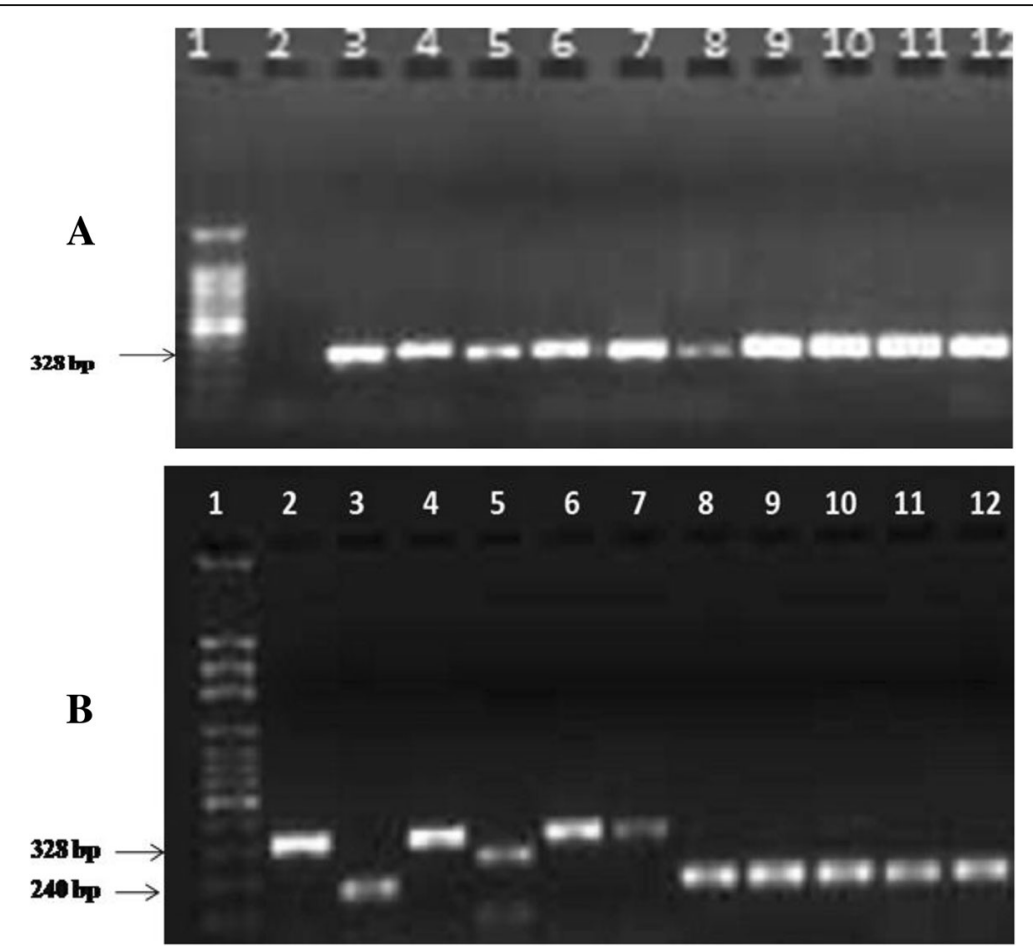

Fig. 2 a PCR products of ITS-1 from promastigote DNA extract, 1. 100 bp ladder, 2. Negative control, 3. L. donovani, 4. L. aethiopica, 5. L. tropica, 6. L. major, 7. L. infantum, 8. L. chagasi, 9-12 clinical isolates. b PCR-ITS1-RFLP of the amplicon with Hha I, 1. 100 bp ladder, 2. L. donovani, 3. L. aethiopica, 4. L. tropica, 5. L. major, 6. L. infantum, 7. L. chagasi, 8-12 clinical isolate

\section{Conclusion}

Our finding indicated that $\mathrm{MCM}$ is a sensitive $\mathrm{CL}$ diagnostic method than TCM and smear microscopy. As it was consistently shown in other studies, MCM also proved to be economical and rapid culturing technique in our finding as well. Thus, this report depicted the value of MCM for the isolation of $L$. aethiopica promastigotes from lesions of $\mathrm{CL}$ patients in Ethiopia.

\section{Abbreviations}

AHRI: Armauer Hansen Research Institute; ALERT: All Africa Leprosy, Tuberculosis and Rehabilitation Training; CL: Cutaneous leishmaniasis; DCL: Diffuse cutaneous leishmaniasis; FBS: Fetal bovine serum; ITS-1: Internal transcriber spacer-1; LCL: Localized cutaneous leishmaniasis;

MCL: Mucocutaneous leishmaniasis; MCM: Microculture method; NNN: NovyMacNeal-Nicolle; PCR: Polymerase chain reaction; RFLP: Restriction fragment length polymorphism; RPMI: Roswell Park Memorial Institute; TCM: Traditional culture method

\section{Acknowledgements}

The authors acknowledge World Health Organization country office for Ethiopia for financing the study and the Armauer Hansen Research Institute (AHRI) for provision of laboratory reagents and consumables for this work.

\section{Funding}

This study was supported by the World Health Organization country office for Ethiopia through Ethiopian Public Health Institute (EPHI); project number AF/ETH/AAC/000/RB/08 AMS CODE: 2123883, and the Armauer Hansen Research Institute (AHRI) core budget.
Availability of data and materials

The datasets generated and analyzed during this study are available from the corresponding author upon reasonable request.

\section{Disclosure statement}

No potential conflict of interest was reported by the authors.

\section{Ethics and consent}

For children 6-18 years of age, the informed consent was obtained from their parents or guardians. An assent was obtained from 12-17 year old children in addition to the consent obtained from their parents or guardians. For all suspected $\mathrm{CL}$ cases, laboratory diagnosis and treatment were given free of charge and all results were kept confidentially.

\section{Authors' contributions}

All authors read and approved the final version of the manuscript. GT and EG conceived the study. GT generated the idea. AA and LA performed the field and laboratory experiments, analyzed the data, and prepared the paper. $A A$ and GT drafted the manuscript. LA, EG, TB, AK, and GT participated on proposal development.

\section{Ethics approval and consent to participate}

This study was ethically approved by Scientific and ethical review Office of Ethiopian Public Health Institute (EI/SERO/SERC/45/2002) and AHRI/ALERT Ethics (PO07/12) committee. Written consent was obtained from all study participants.

\section{Consent for publication}

Not applicable.

\section{Competing interests}

The authors declare that they have no competing interests. 


\section{Publisher's Note}

Springer Nature remains neutral with regard to jurisdictional claims in published maps and institutional affiliations.

\section{Author details}

${ }^{1}$ Armauer Hansen Research Institute, Malaria and Neglected Tropical Diseases Research Directorate, P. O. Box 1005, Addis Ababa, Ethiopia. ${ }^{2}$ Ethiopia Public Health Institute, Malaria and Neglected Tropical Diseases Research Team, P. O. Box 1242, Addis Ababa, Ethiopia. ${ }^{3}$ College of Public and Medical Health, Department of Medical Laboratory and Pathology, Jimma University, P. O. Box 378, Jimma, Ethiopia.

Received: 16 March 2018 Accepted: 30 January 2019 Published online: 21 March 2019

\section{References}

1. Alvar J, Vélez ID, Bern C, Herrero M, Desjeux P, Cano J, et al. Leishmaniasis Worldwide and Global Estimates of Its Incidence. PLoS ONE. 2012;7(5): e35671. https://doi.org/10.1371/journal.pone.0035671.

2. Reithinger R, Dujardin J, Louzir H, Pirmez C, Alexander B, Brooker S, et al. Cutaneous leishmaniasis. Lancet Infect Dis. 2007;7:581-96.

3. MBryceson AD. Immunological aspects of clinical leishmaniasis. Proc roy Soc Med. 1970;63:1056-60.

4. Negera E, Gadisa E, Yamuah L, Engers H, Hussein J, Kuru T, et al. Outbreak of cutaneous leishmaniasis in Silti woreda, Ethiopia : risk factor assessment and causative agent identification. Trans R Soc Trop Med Hyg. 2008;102:883-90.

5. Leishmaniases WHOER of a meeting of the WEC on the $C$ of. Control of the leishmaniases. In: WHO Technical Report Series; 2010. p. 22-6.

6. Hosseinzadeh $\mathrm{M}$, Omidifar $\mathrm{N}$, Lohrasb MH. Short report use of fine needle. Trop Dr. 2012;42(I):112-3.

7. Escobar MA, Martinez F, Scott Smith D, Palma Gl. American cutaneous and mucocutaneous leishmaniasis (tegumentary): a diagnostic challenge. Trop Dr. 1992;22(1):69-78

8. Reithinger R, Dujardin JC. MINIREVIEWS molecular diagnosis of leishmaniasis : current status and future applications. J Clin Microbiol. 2007:45(1):21-5.

9. Mouttaki T, Morales-yuste M, Merino-espinosa G, Chiheb S, Fellah H, Martinsanchez J, et al. Molecular diagnosis of cutaneous leishmaniasis and identification of the causative Leishmania species in Morocco by using three PCR-based assays. Parasit Vectors. 2014:7:1-9.

10. Deborggraeve S, Laurent T, Espinosa D, Van Der Auwera G, Mbuchi M, Wasunna $\mathrm{M}$, et al. MAJOR ARTICLE a simplified and standardized polymerase chain reaction format for the diagnosis of leishmaniasis. J Infect Dis. 2008; 198:1565-72.

11. Boggild AK, Miranda-verastegui C, Espinosa D, Arevalo J, Martinez-medina D, Llanos-cuentas A, et al. Optimization of microculture and evaluation of miniculture for the isolation of leishmania parasites from cutaneous lesions in Peru. Am J Trop Med Hyg. 2008;79(6):847-52.

12. Allahverdiyev AM, Uzun S, Bagirova M, Durdu M, Memisoglu HR. A sensitive new microculture method for diagnosis of cutaneous leishmaniasis. Am J Trop Med Hyg. 2004;70(3):294-7.

13. Hide M, Singh R, Kumar B, Ba AL, Sundar S. Short communication a microculture technique for isolating live leishmania parasites from peripheral blood of visceral leishmaniasis patients. Acta Trop. 2007;102:197-200

14. Pagheh A, Fakhar M. An improved microculture method for diagnosis of cutaneous leishmaniasis. J Parasit Dis. 2014;38(4):347-51.

15. Boggild AK, Ramos AP, Espinosa D, Valencia BM, Veland N, Mirandaverastegui $C$, et al. Clinical and demographic stratification of test performance : a pooled analysis of five laboratory diagnostic methods for American cutaneous leishmaniasis. Am J Trop Med Hyg. 2010;83(2):345-50.

16. Boggild AK, Miranda-verastegui C, Espinosa D, Arevalo J, Adaui V, Tulliano G, et al. Evaluation of a microculture method for isolation of leishmania parasites from cutaneous lesions of patients in Peru. J Clin Microbiol. 2007; 45(11):3680-4.

17. Van Griensven J, Gadisa E, Aseffa A, Hailu A. Treatment of Cutaneous Leishmaniasis Caused by Leishmania aethiopica: A Systematic Review; 2016. p. 1-20.

18. Chulay J, Bryceson A. Quantitation of amastigotes of leishmania donovani in smears of splenic aspirates from patients with visceral leishmaniasis. Am J Trop Med Hyg. 1983;32:475-9.
19. Ihalamulla RL, Rajapaksa US, Siriwardena HVYD, Chance M, Karunaweera ND. A simple, cost effective method for isolation and transportation of Leishmania parasites. Ceylon Med J. 2009;1(January):1-2.

20. Schonian G, Nasereddin A, Dinse N, Schweynoch C, Henk DFH, Schallig WP Jaffe CL. PCR diagnosis and characterization of Leishmania in local and imported clinical samples PCR diagnosis and characterization of Leishmania in local and imported clinical samples 1. Diagn Microbiol Infect Dis. 2003; October:349-58.

21. Gadisa E, Genetu A, Kuru T, Jirata D, Dagne K, Ase AV, et al. Leishmania ( Kinetoplastida ): species typing with isoenzyme and PCR - RFLP from cutaneous leishmaniasis patients in Ethiopia. Exp Parasitol. 2007:115:339-43.

22. Banoo S, Bell D, Bossuyt P, Herring A, Mabey D, et al. Evaluation of diagnostic tests for infectious diseases: general principles. Nat Rev Microbiol. 2006;December:20-32.

23. Niño A, Camacho M. Leishmania (Viannia) braziliensis growth in vitro culture relies more on folic acid availability than leihsmania (Leishmania) amazonensis. Mem Inst Oswaldo Cruz, Rio Janeiro. 2005:100(3):309-10.

24. Serin MS, Daglioglu K, Bagirova M, Allahverdiyev A, Uzun S. Rapid diagnosis and genotyping of leishmania isolates from cutaneous and visceral leishmaniasis by microcapillary cultivation and polymerase chain reaction restriction fragment length polymorphism of miniexon region. Diagn Microbiol Infect Dis. 2005:53:209-14.

25. Tasew G, Kebede A, Wolday D, Gadisa E, Britton S, Eidsmo L, et al. Low-cost liquid medium for in vitro cultivation of Leishmania parasites in low-income countries. Glob Health Action. 2009;1(4):1-5.

26. Croft SL, Shyam Sundar AHF. Drug resistance in Leishmaniasis. Clin Microbiol Rev. 2006;19(1):111-26.

27. Gelanew T, Hurissa Z, Diro E, Kassahun A, Kuhls K, Schönian G, et al. Case report: disseminated cutaneous leishmaniasis resembling post-kala-azar dermal leishmaniasis caused by leishmania donovani in three patients coinfected with visceral leishmaniasis and human immunodeficiency virus/ acquired immunodeficiency syndrome in Ethiopia. Am J Trop Med Hyg. 2011:84(6):906-12
Ready to submit your research? Choose BMC and benefit from:

- fast, convenient online submission

- thorough peer review by experienced researchers in your field

- rapid publication on acceptance

- support for research data, including large and complex data types

- gold Open Access which fosters wider collaboration and increased citations

- maximum visibility for your research: over $100 \mathrm{M}$ website views per year

At BMC, research is always in progress.

Learn more biomedcentral.com/submissions 